IJMMS 25:12 (2001) 777-785

PII. S0161171201004525

http://ijmms.hindawi.com

(c) Hindawi Publishing Corp.

\title{
OPTIMAL PROBLEM OF COST FUNCTION FOR THE LINEAR NEUTRAL SYSTEMS
}

\author{
JONG YEOUL PARK and YONG HAN KANG
}

(Received 24 January 2000)

\begin{abstract}
We study the optimal control problem of a system governed by linear neutral type in Hilbert space $X$. We investigate optimal condition for quadratic cost function and as applications, we give some examples.
\end{abstract}

2000 Mathematics Subject Classification. Primary 93C20, 34K40, 49J25, 49K25.

1. Introduction. Our main concern in this paper is to study the optimal control problem of the linear neutral type:

$$
\begin{aligned}
\frac{d}{d t}\left[x(t)-\sum_{j=1}^{m} B_{j} x\left(t-h_{j}\right)\right] & =A_{0} x(t)+\sum_{j=1}^{m} A_{j} x\left(t-h_{j}\right), \quad t \geq 0, \\
x(0)=g^{0}, \quad x(t) & =g^{1}(t), \quad \text { a.e. } t \in[-h, 0),
\end{aligned}
$$

where $\left(g^{0}, g^{1}\right) \in X \times C([-h, 0] ; X)$.

The optimal control problem of this type has been extensively studied by many authors (see [1, 2] and the references therein). In [2], Darko studied the Laplace transform and fundamental solution in (1.1). Chukwu [1] handled time optimal control, bang-bang control and stability for the neutral type. In fact, in the case of $B_{j}=0, j=$ $1,2, \ldots, m$ in (1.1), Nakagiri [6] studied structural properties of the linear retarded system and dealt with control problems in a Banach spaces.

In this paper, we obtain the necessary and sufficient condition for the optimal control problems of the quadratic cost function and deal with the properties of the fundamental solution and the adjoint state equations in (1.1). As applications, we will give some examples.

2. Preliminaries. (1) $\mathbb{C}$ denotes the complex plane. $\mathbb{R}$ denotes the real numbers, $\mathbb{R}^{+}$ the nonnegative real numbers, and the real interval $[0, T]=I$.

(2) The symbol $X$ denotes a given Banach space over the real. However, in some instances when dealing with Laplace transforms, we have to consider the complex extension of $X$ which will again be denoted by $X$. If $[-h, 0]$ is an interval in $\mathbb{R}$, the Banach space of all continuous mapping $\phi$ from $[-h, 0]$ into $X$ will be denoted by $C([-h, 0] ; X)$. The norm in $C([-h, 0] ; X)$ is defined to be $\|\phi\|=\sup \{|\phi(t)| ; t \in[-h, 0]\}$, where $\|\cdot\|$ denotes the norm in $X$. If $\phi$ in $C([-h, 0] ; X)$ has a derivative which is also in $C([-h, 0] ; X)$, this will be denoted by $(d / d t) \phi(t)=\dot{\phi}(t)$. 
(3) If $Y$ is a given Banach space, the space of continuous linear mappings from $Y$ into itself will be denoted by $L C(Y)$. If $Z$ is also Banach space, the space of all continuous linear mappings from $Y$ into $Z$ will be denoted by $L C(Y, Z)$.

(4) If $X$ and $Y$ be a given Banach space, the space of bounded mappings from $X$ into $Y$ will be denoted by $B(X, Y)$ and if $X=Y$, by $B(X)$.

(5) Let $A_{0}: X \rightarrow X$ be a closed linear operator which is the infinitesimal generator of a semigroup, $T(t)=e^{A_{0} t}$, of class $C_{0}$ on $X$. The domain, $\mathscr{D}\left(A_{0}\right)$, of $A_{0}$ is dense in $X$ (cf. [3]) and since $T(t)$ is of class $C_{0}$ there exist constants $M \geq 1$ and $\alpha$ such that $\|T(t)\| \leq M e^{\alpha t}$ (again see [3]).

(6) $\left\{A_{j}\right\}$ and $\left\{B_{j}\right\}, 1 \leq j \leq m$, are operators in $L C(X)$, for each $j$ we assume range $\left(B_{j}\right)$ is in $\mathscr{D}\left(A_{0}\right)$ for each $j$ and $B \in L_{\infty}([0, T] ; X)$.

(7) The numbers $0<h_{1}<h_{2}<h_{3}<\cdots<h_{m}=h$ are fixed in $\mathbb{R}$.

Under the above hypotheses, we consider an "integrated" form described by the equations in (1.1);

$$
\begin{aligned}
x\left(t, 0,\left(g^{0}, g^{1}\right)\right)= & \sum_{j=1}^{m} B_{j} x\left(t-h_{j}, 0, g\right)+T(t)\left[g^{0}-\sum_{j=1}^{m} B_{j} g^{1}\left(-h_{j}\right)\right] \\
& +\int_{0}^{t} T(t-\sigma)\left[\sum_{j=1}^{m}\left(A_{j}+A_{0} B_{j}\right) x\left(\sigma-h_{j}, 0, g\right)\right] d \sigma, \quad t \geq 0, \\
x(t, 0, g)= & g^{1}(t), \quad \text { a.e. } t \in[-h, 0],
\end{aligned}
$$

where $g=\left(g^{0}, g^{1}\right) \in X \times C([-h, 0] ; X)$.

Here, $x(t, 0, g)$ is the solution with initial condition $t=0$.

Note that if $f \in C(I ; X)$, then

$$
\frac{d}{d t} \int_{0}^{t} T(t-\sigma) f(\sigma) d \sigma=A_{0} \int_{0}^{t} T(t-\sigma) f(\sigma) d \sigma+f(t)
$$

(cf. [2]). Thus we can differentiate (2.1) to obtain

$$
\begin{aligned}
\frac{d}{d t} x(t, 0, g)= & \sum_{j=1}^{m} B_{j} \frac{d}{d t} x\left(t-h_{j}, 0, g\right)+\frac{d}{d t}\left[T(t)\left(g^{0}-\sum_{j=1}^{m} B_{j} g^{1}\left(-h_{j}\right)\right)\right] \\
& +\sum_{j=1}^{m}\left(A_{j}+A_{0} B_{j}\right) x\left(t-h_{j}, 0, g\right) \\
& +A_{0} \int_{0}^{t} T(t-\sigma)\left[\sum_{j=1}^{m}\left(A_{j}+A_{0} B_{j}\right) x\left(\sigma-h_{j}, 0, g\right)\right] d \sigma .
\end{aligned}
$$

Since $(d / d t)\left(T(t) g^{0}\right)=A_{0} T(t) g^{0}$, if the derivative exists, and making use of (2.1) we again obtain from (2.3) the equation

$$
\begin{aligned}
\dot{x}(t, 0, g)-\sum_{j=1}^{m} B_{j} \dot{x}\left(t-h_{j}, 0, g\right)= & A_{0}\left(x(t, 0, g)-\sum_{j=1}^{m} B_{j} x\left(t-h_{j}, 0, g\right)\right) \\
& +\sum_{j=1}^{m} A_{j} x\left(t-h_{j}, 0, g\right)+A_{0} \sum_{j=1}^{m} B_{j} x\left(t-h_{j}, 0, g\right) \\
= & A_{0} x(t, 0, g)+\sum_{j=1}^{m} A_{j} x\left(t-h_{j}, 0, g\right)
\end{aligned}
$$

(cf. [2, Theorem 2]). 
3. Optimality conditions for quadratic cost function. First of all, we consider the construction of the solution in the following type:

$$
\begin{gathered}
\frac{d}{d t}\left[x(t)-\sum_{j=1}^{m} B_{j} x\left(t-h_{j}\right)\right]=A_{0} x(t)+\sum_{j=1}^{m} A_{j} x\left(t-h_{j}\right)+B(t) u(t), \quad t \geq 0 \\
x(0)=g^{0}, \quad x(t)=g^{1}(t), \quad \text { a.e. } t \in[-h, 0),
\end{gathered}
$$

where $\left(g^{0}, g^{1}\right) \in X \times C([-h, 0] ; X)$.

Define the fundamental solution $W(t)$ of (3.1) by

$$
W(t) g^{0}= \begin{cases}x\left(t ; 0,\left(g^{0}, 0\right)\right), & t \geq 0 \\ 0, & t<0\end{cases}
$$

where $x\left(t ; u,\left(g^{0}, g^{1}\right)\right)$ is the general solution of (3.1) (see [1]).

Hence $W(t)$ is the unique solution of

$$
W(t)=T(t)+\sum_{j=1}^{m} \chi\left(t-h_{j}\right) B_{j} W\left(t-h_{j}\right)+\int_{0}^{t} T(t-\sigma) \sum_{j=1}^{m} \chi\left(\sigma-h_{j}\right)\left(A_{j}+A_{0} B_{j}\right) W\left(\sigma-h_{j}\right) d \sigma,
$$

where $\chi(\sigma)=0$ if $\sigma<0, \chi(\sigma)=I$ if $\sigma \geq 0, I$ identity (cf. [1]).

Note that if $g^{1} \in C([-h, 0] ; X)$ is absolutely continuous, then the solution of (3.1) can be written as

$$
\begin{aligned}
x\left(t ; u,\left(g^{0}, g^{1}\right)\right)= & {\left[W(t)-\sum_{j=1}^{m} W\left(t-h_{j}\right) B_{j}\right] g^{0} } \\
& +\sum_{j=1}^{m} \int_{h_{j}}^{0} W\left(t-s-h_{j}\right)\left[A_{j} g^{1}(s)+B_{j} g^{1}(s)\right] d s \\
& +\int_{0}^{t} W(t-s) B(s) u(s) d s \\
= & x\left(t ; 0,\left(g^{0}, g^{1}\right)\right)+\int_{0}^{t} W(t-s) B(s) u(s) d s .
\end{aligned}
$$

(Cf. [4, page 400]).

In the following, we obtain the properties of the fundamental solution.

LEMMA 3.1. Let $W(t)$ be fundamental solution of (3.1). Then we have the following:

$$
\frac{d}{d t}\left[W(t)-\sum_{j=1}^{m} B_{j} W\left(t-h_{j}\right)\right]=A_{0} W(t)+\sum_{j=1}^{m} A_{j} W\left(t-h_{j}\right)
$$


Proof. From (3.3) and $(d / d t) T(t)=A_{0} T(t)$,

$$
\begin{aligned}
\frac{d}{d t}\left[W(t)-\sum_{j=1}^{m} \chi\left(t-h_{j}\right) B_{j} W\left(t-h_{j}\right)\right] \\
=\frac{d}{d t}\left[T(t)+\int_{0}^{t} T(t-s) \sum_{j=1}^{m} \chi\left(s-h_{j}\right)\left(A_{j}+A_{0} B_{j}\right) W\left(s-h_{j}\right) d s\right] \\
=A_{0} T(t)+\int_{0}^{t} A_{0} T(t-s) \sum_{j=1}^{m} \chi\left(s-h_{j}\right)\left(A_{j}+A_{0} B_{j}\right) W\left(s-h_{j}\right) d s \\
\quad+\sum_{j=1}^{m} \chi\left(t-h_{j}\right)\left(A_{j}+A_{0} B_{j}\right) W\left(t-h_{j}\right) \\
=A_{0}\left[T(t)+\int_{0}^{t} T(t-s) \sum_{j=1}^{m} \chi\left(s-h_{j}\right)\left(A_{j}+A_{0} B_{j}\right) W\left(s-h_{j}\right) d s\right] \\
\quad+\sum_{j=1}^{m} x\left(t-h_{j}\right)\left(A_{j}+A_{0} B_{j}\right) W\left(t-h_{j}\right) \\
=A_{0} W(t)-A_{0} \sum_{j=1}^{m} \chi\left(t-h_{j}\right) B_{j} W\left(t-h_{j}\right)+\sum_{j=1}^{m} \chi\left(t-h_{j}\right)\left(A_{j}+A_{0} B_{j}\right) W\left(t-h_{j}\right) \\
=A_{0} W(t)+\sum_{j=1}^{m} \chi\left(t-h_{j}\right) A_{j} W\left(t-h_{j}\right) .
\end{aligned}
$$

Since definition of $\chi(\cdot)$,

$$
\begin{aligned}
& \sum_{j=1}^{m} \chi\left(t-h_{j}\right) B_{j} W\left(t-h_{j}\right)=\sum_{j=1}^{m} B_{j} W\left(t-h_{j}\right), \\
& \sum_{j=1}^{m} \chi\left(t-h_{j}\right) A_{j} W\left(t-h_{j}\right)=\sum_{j=1}^{m} A_{j} W\left(t-h_{j}\right) .
\end{aligned}
$$

Hence this proof is complete.

$W^{*}(t), A_{j}^{*}, A_{0}^{*}$, and $B_{j}^{*}$ denote the adjoint operators of $W(t), A_{j}, A_{0}$, and $B_{j}$, respectively.

A similar method as in Lemma 3.1, we consider Lemma 3.2.

LEMMA 3.2. Let $W(t)$ be fundamental solution of (3.1). Then

$$
\frac{d}{d t}\left(W^{*}(t)-\sum_{j=1}^{m} W^{*}\left(t-h_{j}\right) B_{j}^{*}\right)=A_{0}^{*} W^{*}(t)+\sum_{j=1}^{m} \chi\left(t-h_{j}\right) A_{j}^{*} W^{*}\left(t-h_{j}\right) .
$$

Proof. From (3.3) and $A_{0}^{*} W^{*}(t)=W^{*}(t) A_{0}^{*}, A_{j}^{*} W^{*}(t)=W^{*}(t) A_{j}^{*}, B_{j}^{*} W^{*}(t)=$ $W^{*}(t) B_{j}^{*}$, we have

$$
W^{*}(t)-\sum_{j=1}^{m} \chi\left(t-h_{j}\right) B_{j}^{*} W^{*}\left(t-h_{j}\right)=T^{*}(t)+\int_{0}^{t} T^{*}(t-\sigma) \sum_{j=1}^{m}\left(A_{j}^{*}+A_{0}^{*} B_{j}^{*}\right) W^{*}\left(\sigma-h_{j}\right) d \sigma .
$$


Differenting (3.9) and using $(d / d t) T^{*}(t)=A_{0}^{*} T^{*}(t)$, we have

$$
\begin{aligned}
\frac{d}{d t}[ & \left.W^{*}(t)-\sum_{j=1}^{m} \chi\left(t-h_{j}\right) B_{j}^{*} W^{*}\left(t-h_{j}\right)\right] \\
= & A_{0}^{*} T^{*}(t)+\sum_{j=1}^{m} \chi\left(t-h_{j}\right)\left(A_{j}^{*}+A_{0}^{*} B_{j}^{*}\right) W^{*}\left(t-h_{j}\right) \\
& +\int_{0}^{t} A_{0}^{*} T^{*}(t-\sigma) \sum_{j=1}^{m} \chi\left(\sigma-h_{j}\right)\left(A_{j}^{*}+A_{0}^{*} B_{j}^{*}\right) W^{*}\left(\sigma-h_{j}\right) d \sigma \\
= & A_{0}^{*}\left[T^{*}(t)+\int_{0}^{t} T^{*}(t-\sigma) \sum_{j=1}^{m} \chi\left(\sigma-h_{j}\right)\left(A_{j}^{*}+A_{0}^{*} B_{j}^{*}\right) W^{*}\left(\sigma-h_{j}\right) d \sigma\right] \\
& +\sum_{j=1}^{m} \chi\left(t-h_{j}\right)\left(A_{j}^{*}+A_{0}^{*} B_{j}^{*}\right) W^{*}\left(t-h_{j}\right) \\
= & A_{0}^{*}\left[W^{*}(t)-\sum_{j=1}^{m} \chi\left(t-h_{j}\right) B_{j}^{*} W^{*}\left(t-h_{j}\right)\right] \\
& +\sum_{j=1}^{m} \chi\left(t-h_{j}\right)\left(A_{j}^{*}+A_{0}^{*} B_{j}^{*}\right) W^{*}\left(t-h_{j}\right) \\
= & A_{0}^{*} W^{*}(t)+\sum_{j=1}^{m} \chi\left(t-h_{j}\right) A_{j}^{*} W^{*}\left(t-h_{j}\right) .
\end{aligned}
$$

Since definition of $\chi(\cdot)$,

$$
\begin{aligned}
& \sum_{j=1}^{m} \chi\left(t-h_{j}\right) B_{j}^{*} W^{*}\left(t-h_{j}\right)=\sum_{j=1}^{m} B_{j}^{*} W^{*}\left(t-h_{j}\right), \\
& \sum_{j=1}^{m} \chi\left(t-h_{j}\right) A_{j}^{*} W^{*}\left(t-h_{j}\right)=\sum_{j=1}^{m} A_{j}^{*} W^{*}\left(t-h_{j}\right) .
\end{aligned}
$$

Hence this proof is complete.

Secondly, we consider the following cost function:

$$
\mathscr{g}(u)=\int_{0}^{T}\left\|C x_{u}(t)-z_{d}\right\|_{X}^{2} d t+\int_{0}^{T}(N u(t), u(t)) d t
$$

where the observation operator $C$ is bounded from $H$ to another Hilbert space $X$, every control $u \in L^{2}(0, T ; U)$ and $z_{d} \in L^{2}(I ; X), I=[0, T]$.

Finally, we assume that $N$ is a selfadjoint operator in $B(X)$ such that

$$
(N u, u) \geq c\|u\|^{2}, \quad c>0
$$

where $B(X)$ denotes the space of bounded operators on $X$. Let $x_{u}(t)$ stands for a solution of (3.1) associated with the control $u \in L^{2}(0, T ; U)$. Let $U_{\text {ad }}$ be a closed convex subset of $L^{2}(0, T ; U)$. 
Theorem 3.3. Let the operators $C$ and $N$ satisfy conditions (3.12) and (3.13). Then there exists a unique element $u \in U_{\text {ad }}$ such that

$$
\mathscr{L}(u)=\inf _{v \in U_{\mathrm{ad}}} \mathscr{F}(v) .
$$

Furthermore, it is hold the following inequality:

$$
\int_{0}^{T}\left(-\Lambda^{-1} B(t)^{*} p(s)+N(s), v(s)-u(s)\right) d s \geq 0,
$$

where $p(s)$ is a solution of adjoint state equation for (3.1) and with the initial condition $p(s)=0$ for $s \in[T, T+h]$ substituting $q_{1}^{*}$ by $-C^{*} \Lambda\left(C x_{u}(t)-z_{d}\right)$. That is, $p(t)$ satisfies the following transposed system:

$$
\begin{gathered}
\frac{d}{d t} p(t)+\sum_{j=1}^{m} B_{j}^{*} \frac{d}{d t} p\left(t+h_{j}\right)+A_{0}^{*} p(t)+\sum_{j=1}^{m} A_{j}^{*} p\left(t+h_{j}\right)+C^{*} \Lambda\left(z_{d}-C x_{u}(t)\right)=0 \text {, a.e. } t \in I, \\
p(s)=0 \text { a.e. } s \in[T, T+h]
\end{gathered}
$$

in the weak sense. Here, the operator $\Lambda$ is the canonical isomorphism of $U$ onto $U^{*}$.

Proof. Let $x(t)=x\left(t ; 0,\left(g^{0}, g^{1}\right)\right)$. Then it holds that

$$
\begin{aligned}
\mathscr{g}(v) & =\int_{0}^{T}\left\|C x_{v}(t)-z_{d}\right\|^{2} d t+\int_{0}^{T}(N v(t), v(t)) d t \\
& =\int_{0}^{T}\left\|C\left(x_{v}(t)-x(t)\right)+C x(t)-z_{d}\right\|^{2} d t+\int_{0}^{T}(N v(t), v(t)) d t \\
& =\pi(u, v)-2 L(v)+\int_{0}^{T}\left\|z_{d}-C x(t)\right\|^{2} d t
\end{aligned}
$$

where

$$
\begin{aligned}
\pi(u, v) & =\int_{0}^{T}\left(C\left(x_{u}(t)-x(t)\right), C\left(x_{v}(t)-x(t)\right)\right) d t+\int_{0}^{T}(N u(t), v(t)) d t \\
L(v) & =\int_{0}^{T}\left(z_{d}-C x(t), C\left(x_{v}(t)-x(t)\right)\right) d t
\end{aligned}
$$

The form $\pi(u, v)$ is a continuous bilinear form in $L^{2}(0, T ; U)$ and from the assumption that the operator $N$ is positive definite, we have

$$
\pi(v, v) \geq c\|v\|^{2}, \quad v \in L^{2}(0, T ; U) .
$$

Therefore in virtue of Theorem 1.1 of Chapter 1 in [5], there exists a unique $u \in$ $L^{2}(0, T ; U)$ such that (3.14) holds.

If $u$ is an optimal control (cf. [5, Theorem 1.3. of Chapter 1]), then

$$
\mathscr{g}^{\prime}(u)(v-u) \geq 0, \quad u \in U_{\mathrm{ad}},
$$


where $\mathscr{g}^{\prime}(u) v$ means the Frechet derivative of $\mathscr{F}$ at $u$, applied to $v$

$$
\begin{aligned}
\mathscr{g}^{\prime}(u)(v-u)= & \int_{0}^{T}\left(C x_{u}(t)-z_{d}, C \int_{0}^{t} W(t-s) B(s)(v(s)-u(s))\right) d s d t \\
& +\int_{0}^{T}(N u(t), v(t)-u(t)) d t \\
= & \int_{0}^{T}\left(C^{*} \Lambda\left(C x_{u}(t)-z_{d}\right), \int_{0}^{t} W(t-s) B(s)(v(s)-u(s)) d s\right) d t .
\end{aligned}
$$

Note that $C^{*} \in B\left(X^{*}, H\right)$ and for $\phi$ and $\psi$ in $H$, we have

$$
\left(C^{*} \Lambda C \psi, \phi\right)=(C \psi, C \phi)
$$

where duality pairing is also denoted by $(\cdot, \cdot)$.

From Fubini's theorem, we have

$$
\begin{aligned}
\int_{0}^{T} \int_{0}^{t} & \left(C^{*} \Lambda\left(C x_{u}(t)-z_{d}\right), W(t-s) B(s)(v(s)-u(s))\right) d s d t+\int_{0}^{T}(N u(t), v(t)-u(t)) d t \\
= & \int_{0}^{T} \int_{s}^{T}\left(C^{*} \Lambda\left(C x_{u}(t)-z_{d}\right), W(t-s) B(s)(v(s)-u(s))\right) d t d s \\
& \quad+\int_{0}^{T}(N u(t), v(t)-u(t)) d t \\
= & \int_{0}^{T}\left(\int_{s}^{T}\left(\Lambda^{-1} B^{*}(s) W^{*}(t-s) C^{*} \Lambda\left(C x_{u}(t)-z_{d}\right)\right) d t+N u(s), v(s)-u(s)\right) d s \\
= & \int_{0}^{T}\left(-\Lambda^{-1} B^{*}(s) p(s)+N u(s), v(s)-u(s)\right) d s \geq 0
\end{aligned}
$$

where $p(s)$ is given by (3.14) and (3.16), that is,

$$
p(s)=-\int_{s}^{T} W^{*}(t-s) C^{*} \Lambda\left(C x_{u}(t)-z_{d}\right) d t .
$$

By using Lemma 3.2 and differentiating (3.25) with respect to $s$, we get (3.16).

COROllary 3.4 (maximal principle). Let $u$ be an optimal solution for $\mathscr{F}$. Then

$$
\max _{v \in U_{\mathrm{ad}}}\left(v, \Lambda^{-1} B^{*}(s) p(s)\right)=\left(u, \Lambda^{-1} B^{*}(s) p(s)\right),
$$

where $p(s)$ is as in Theorem 3.3.

In application, by using Lemmas 3.1 and 3.2, Theorem 3.3, and differentiating $p(s)$ with respect to $s$, we obtain some examples.

The cost $\mathscr{F}_{1}$ is given by

$$
\mathscr{g}_{1}=\left\langle x(T), \psi_{0}^{*}\right\rangle+\int_{I}\left\langle x(t), \psi_{1}^{*}(t)\right\rangle d t,
$$

where $\psi_{0}^{*} \in X^{*}$ and $\psi_{1}^{*} \in L_{1}\left(I ; X^{*}\right)$.

Then we have the following example. 
EXAMPLE 3.5 (special linearized Bolza problem). Let $(u, x) \in U_{\mathrm{ad}} \times C(I ; X)$ be an optimal solution for $\mathscr{f}_{1}$ in (3.27). Then

$$
\max _{v \in U(t)}\langle B(t) v, p(t)\rangle=\langle B(t) u(t), p(t)\rangle \quad \text { a.e. } t \in I,
$$

where

$$
p(t)=-W^{*}(T-t) \psi_{0}^{*}-\int_{t}^{T} W^{*}(s-t) \psi^{*}(s) d s, \quad t \in I .
$$

If $X$ is reflexive, then $p(t)$ in (3.29) belongs to $C\left(I ; X^{*}\right)$ and satisfies

$$
\begin{gathered}
\frac{d}{d t}\left[p(t)+\sum_{j=1}^{m} B_{j}^{*} p\left(t-h_{j}\right)\right]+A_{0} p(t)+\sum_{j=1}^{m} A_{j}^{*} p\left(t+h_{j}\right)-\psi_{1}^{*}(t)=0 \quad \text { a.e. } t \in I, \\
p(T)=-\psi_{0}^{*}, \quad p(s)=0, \quad s \in(T, T+h]
\end{gathered}
$$

in the weak sense.

Let $X$ be a Hilbert space. As usual we identify $X$ and $X^{*}$. The cost $\mathscr{f}_{2}$ is given by

$$
\mathscr{g}_{2}=\frac{1}{2}\left|x(T)-x_{d}\right|^{2}, \quad x_{d} \in X
$$

EXAMPLE 3.6 (terminal value control problem). Let $(u, x) \in U_{\mathrm{ad}} \times C(I ; X)$ be an optimal solution for $\mathscr{F}_{2}$ in (3.31). Then

$$
\max _{v \in U(t)}(B(t) v, p(t))=(B(t) u(t), p(t)) \quad \text { a.e. } t \in I,
$$

where $p(t)$ is given by

$$
p(t)=W^{*}(T-t)\left(x_{d}-x(T)\right), \quad t \in I .
$$

The adjoint state $p \in C(I ; X)$ in (3.33) satisfies

$$
\begin{gathered}
\frac{d}{d t}\left[p(t)+\sum_{j=1}^{m} B_{j=1}^{*} p\left(t+h_{j}\right)\right]+A_{0}^{*} p(t)+\sum_{j=1}^{m} A_{j}^{*} p\left(t+h_{j}\right)=0 \quad \text { a.e. } t \in I, \\
p(T)=x_{d}-x(T), \quad p(s)=0, \quad s \in(T, T+h]
\end{gathered}
$$

in the weak sense.

Let $X$ and $Y$ be Hilbert spaces. The cost $\mathscr{F}_{3}$ is given by

$$
\mathscr{F}_{3}=\int_{I}\left(\lambda^{2}|x(t)|^{2}+|u(t)|_{Y}^{2}\right) d t,
$$

where $\lambda>0$. Then we have the following example.

EXAMPLE 3.7 (minimum energy problem). Let $(u, x) \in U_{\mathrm{ad}} \times C(I ; X)$ be an optimal solution for $\mathscr{f}_{3}$ in (3.35). Then

$$
\max _{v \in U(t)}\left((B(t) v, p(t))-|v|_{Y}^{2}\right)=(B(t) u(t), p(t))-|u(t)|_{Y}^{2} \quad \text { a.e. } t \in I,
$$


where

$$
p(t)=-\int_{t}^{T} W^{*}(s-t)\left(2 \lambda^{2} x(s)\right) d s, \quad t \in I .
$$

The adjoint state $p \in C(I ; X)$ in (3.37) satisfies

$$
\begin{gathered}
\frac{d}{d t}\left[p(t)+\sum_{j=1}^{m} B_{j} p\left(t+h_{j}\right)\right]+A_{0}^{*} p(t)+\sum_{j=1}^{m} A_{j}^{*} p\left(t+h_{j}\right)-2 \lambda^{2} x(t)=0 \quad \text { a.e. } t \in I, \\
p(s)=0, \quad s \in[T, T+h]
\end{gathered}
$$

in the weak sense.

\section{REFERENCES}

[1] E. N. Chukwu, Stability and Time-optimal Control of Hereditary Systems, Mathematics in Science and Engineering, vol. 188, Academic Press, Boston, MA, 1992. MR 93d:49003. Zbl 751.93067.

[2] R. Darko, Linear autonomous neutral differential equations in a Banach space, J. Diff. Equations 25 (1977), 258-274.

[3] E. Hille and R. S. Phillips, Functional Analysis and Semi-groups, American Mathematical Society Colloquium Publications, vol. 31, American Mathematical Society, Rhode Island, 1957, revised edition. MR 19,664d. Zbl 078.10004.

[4] J. M. Jeong and J. G. Kim, Optimality conditions for functional differential equations with time delay, Korea Pusan Kyongnam Math. J. 9 (1993), no. 2, 352-363.

[5] J. L. Lions, Optimal Control of Systems Governed by Partial Differential Equations, Translated from the French by S. K. Mitter. Die Grundlehren der mathematischen Wissenschaften, vol. 170, Springer-Verlag, New York, Berlin, 1971. MR 42\#6395. Zbl 203.09001.

[6] S. Nakagiri, Optimal control of linear retarded systems in Banach spaces, J. Math. Anal. Appl. 120 (1986), no. 1, 169-210. MR 88h:49037. Zbl 603.49005.

Jong Yeoul Park: Department of Mathematics, Pusan National University, Pusan 609-735, KOREA

E-mail address: jyepark@hyowon. pusan.ac.kr

Yong Han Kang: Department of Mathematics, Pusan National University, Pusan 609-735, KOREA 


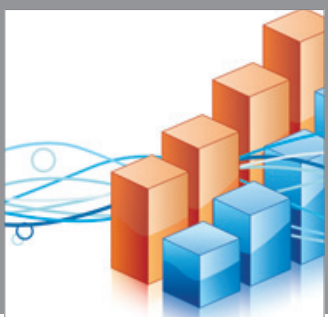

Advances in

Operations Research

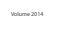

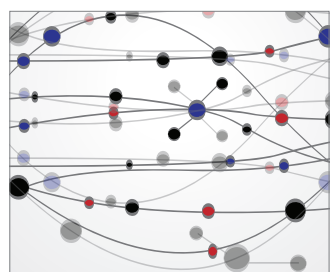

\section{The Scientific} World Journal
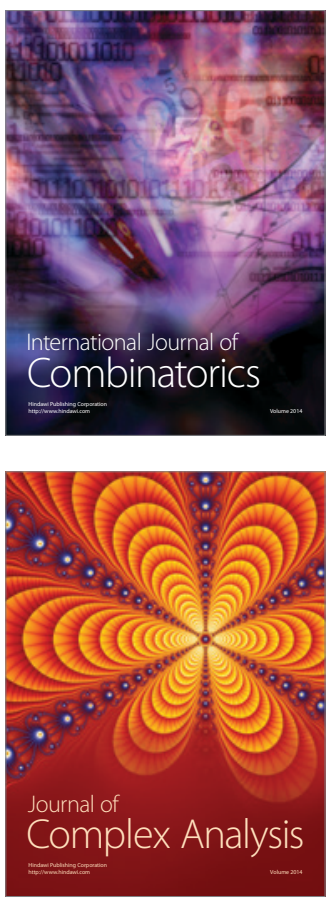

International Journal of

Mathematics and

Mathematical

Sciences
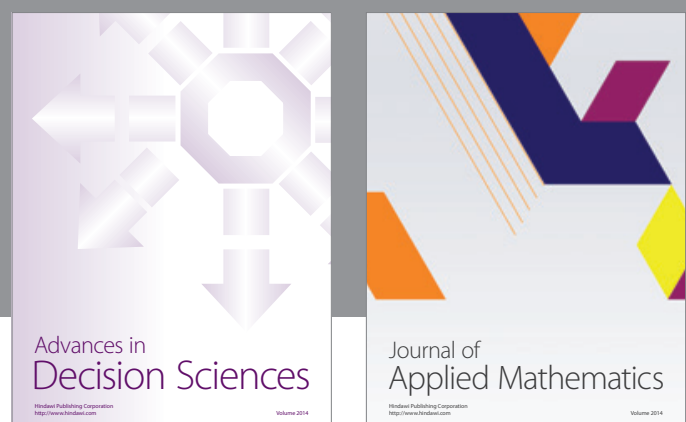

Journal of

Applied Mathematics
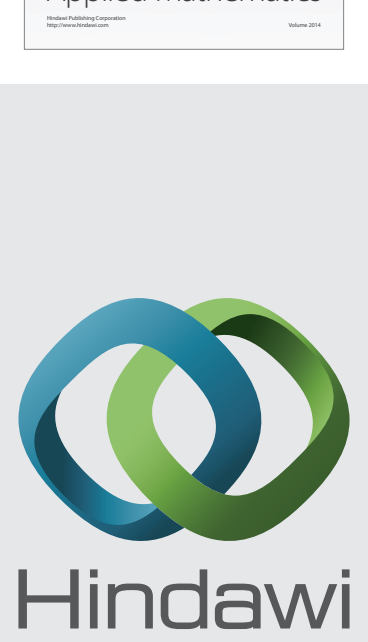

Submit your manuscripts at http://www.hindawi.com
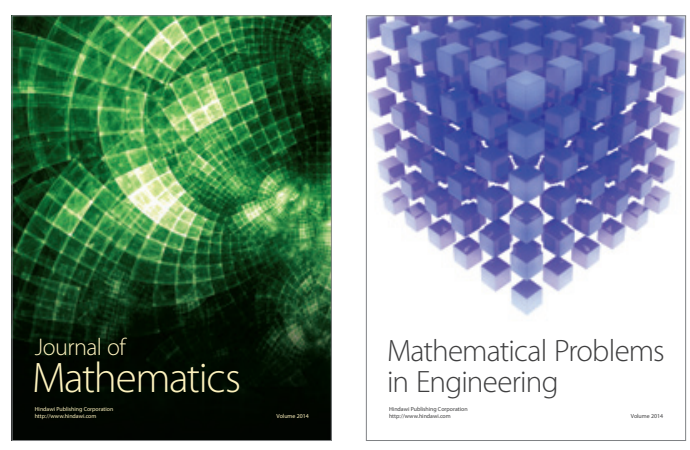

Mathematical Problems in Engineering
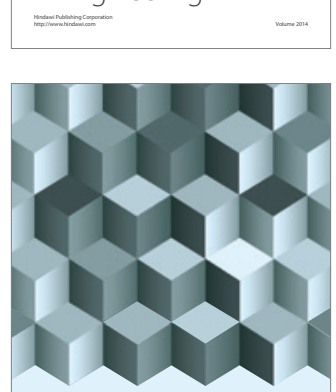

Journal of

Function Spaces
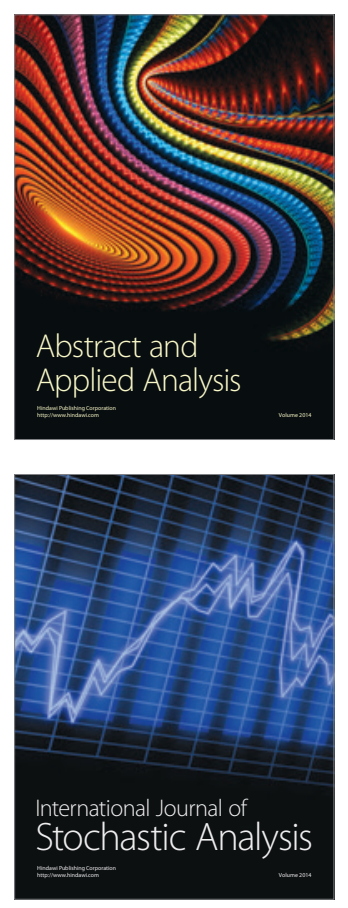

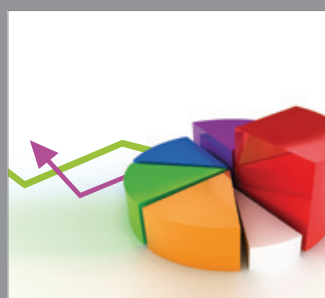

ournal of

Probability and Statistics

Promensencen
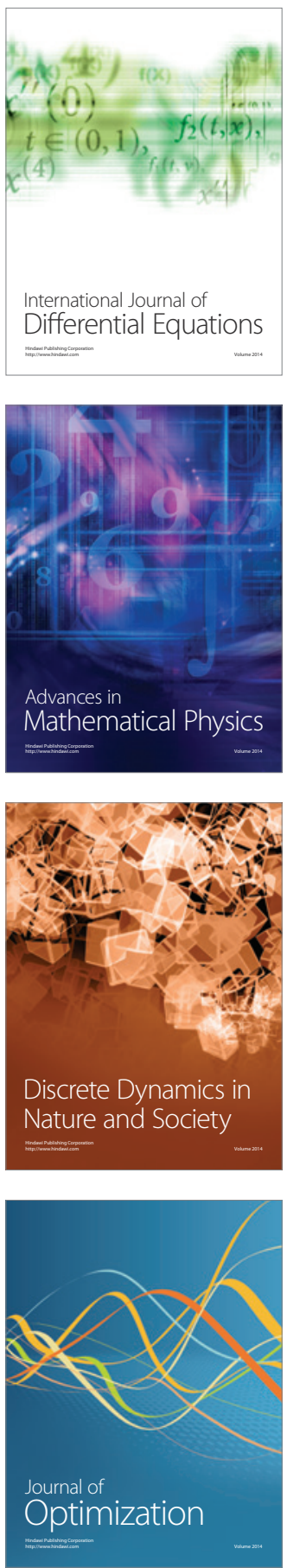